\title{
The Stability of Neuropsychological Test Performance in a Group of Parenteral Drug Users
}

\author{
M. Richards, PhD, M. Sano, PhD, S. Goldstein, ma, \\ D. MindRy, BS, G. TodaK, CSW, ANd Y. STERn, PhD

\begin{abstract}
HIV Center for Clinical and Behavioral Studies, New York State Psychiatric Institute, and the
Departments of Neurology and Psychiatry of the College of Physicians and Surgeons of Columbia University
\end{abstract}

\begin{abstract}
The stability of neuropsychological performance in a sample of drug abusers was investigated for a wide range of neuropsychological tests, using a test-retest paradigm with 16 parenteral drug users. The battery administered included tests of general intellectual function, abstract reasoning, verbal memory, language, attention, visuospatial ability, set switching, speeded performance, and manipulative dexterity. Stability coefficients were of a moderate to high magnitude for most of the tests and were comparable to coefficients found in other studies of non-drug-users. Two exceptions, however, were the Selective Reminding Test and the Perdue Pegboard. Possible reasons for instability with these two tests are discussed. It is concluded that neuropsychological investigations of drug abusers can yield consistent and reliable data, although further studies should employ alternative and/or supplementary measures of verbal memory and motor function.
\end{abstract}

Keywords-test-retest; reliability; stability; neuropsychology; parenteral.

\section{INTRODUCTION}

THE EFFECT OF SUBSTANCE ABUSE On cognitive and motor function has long been of concern to neuropsychologists. While many investigators have focused on deficits among alcoholics, there has been considerable interest in the neuropsychological performance of drug abusers, beginning with the early studies of Brown and Partington (1942). This interest has been reinforced by the recognition that parenteral drug abusers are a principal vector for the transmission of Human Immunodeficiency Virus (HIV) and are thus a major target group for Acquired Immune Deficiency Syndrome (AIDS). It is likely, therefore, that research in this area will continue to be an important focus. However, there are several potential reasons for caution regarding the

C C This research was supported by Center grant MH-43520 from the National Institute of Mental Health and The National Institute on Drug Abuse (Anke A. Ehrhardt, PhD, Principal Investigator).

The authors wish to acknowledge the contribution of Joan McKinnon, RN.

C) Requests for reprints should be addressed to Yaakov Stern PhD, College of Physicians \& Surgeons of Columbia University, Department of Neurology, 710 West 168 Street, New York, NY 10032. stability and consistency of neuropsychological test scores over time with this population. Variations in performance might result from acute drug toxicity and from bchavioral consequences of toxicity, such as disturbances in sleep. In addition, fluctuations in motivation and compliance during testing may arise from long-term changes in personality resulting from chronic patterns of drug abuse.

It is important to assess the effects of acute drug toxicity on the consistency of neuropsychological test scores for the simple reason that the internal drug levels of drug addicts are unlikely to be constant over time. Purity and availability of illicit drugs, especially opiates, may fluctuate, and episodic patterns of use are common with some drugs. Cocaine, in particular, is frequently the object of "bingeing," that is, the consumption of large quantities during a single 48-hour period (e.g., a weekend), followed by a period of abstinence or "crash" (American Psychiatric Association, 1987). These sources of variance in drug levels are reduced among addicts undergoing methadone therapy, since the methadone dose for these individuals is adjusted until a stabilization level is reached. The patient is then maintained at this level on a daily basis. However, even 
with this group, sporadic lapses into illicit drug use occur (e.g., Cheny, Roszell, \& Cummings, 1982; Judson \& Goldstein, 1983; Roszell \& Calsyn, 1986).

What are the effects of acute drug toxicity on neuropsychological performance? Guerra, Sole, Cami, and Tobena (1987) found no differences in measures of perception, attention, verbal fluency, verbal memory or nonverbal intelligence between subjects who admitted to being under the effects of an opiate during testing and subjects who declared themselves to be drug-free during the hours prior to testing. The authors note, however, that the subjective reports of drug state were not corroborated by urinalysis. Yet Rounsaville, Jones, Novelly, and Kleber (1982) found no evidence of drug effects on a variety of neuropsychological tests during an initial assessment when urine screens themselves were used to differentiate drug states (although subjects who tested positive for opiates during a follow-up examination had a stronger grip strength than those who were drug-free). It should be noted that these authors postponed testing for subjects who showed behavioral signs of drug toxicity. It is likely, therefore, that differences between subjects who were intoxicated and those who were drug-free during testing were reduced by this safeguard. However, the same precaution was taken by Grant, Adams, Carlin, Rennick, Judd, and Schooff (1978), who did find a significant elevation in neuropsychological impairment among subjects who tested positive for drugs (barbiturates, amphetamines, minor tranquilizers, or opiates). Unfortunately, these authors do not specify which individual tests were affected.

In addition to the effects of acute toxicity, temporal instability in the neuropsychological performance of drug abusers may arise from long-term changes in personality associated with chronic drug use. While there is some disagreement over the direction of causation between substance abuse and psychopathology (Meyer \& Hesselbrock, 1984), many authors have reported an elevated instance of personality disorder among drug abusers (e.g., Geurra et al., 1987; Grant et al., 1978; Ross, Glaser, \& Germanson, 1988; Rounsaville, Weissman \& Wilber, 1982; Weiss \& Mirin, 1984). It is reasonable, therefore, to anticipate unpredictable neuropsychological performance with this population resulting from variations in compliance and motivation during the test situation, or from different experimenter-subject interactions where more than one tester is employed. Such effects may be reduced among drug users enrolled in a treatment program, where a favorable motivational set can be engendered (Grant et al., 1978). However, they are a potential source of unreliability in the test scores of untreated drug abusers. Few data are available to clarify this complex issue, although one study gives grounds for optimism: Wagner and Caldwell (1979) found high test-retest reliability with the WAIS among outpatients diagnosed as having a personality disorder. It should be noted, however, that these patients were not drug abusers and so the question is not directly addressed.

In sum, acute drug toxicity and psychopathological changes associated with long-term drug abuse are potential sources of inconsistency when assessing neuropsychological function in drug users. Where these factors are uncontrolled (and, in the case of untreated polydrug abusers, they can be very difficult to control), they are likely to inflate the error variance in both crosssectional and longitudinal studies. In spite of this, there have been few attempts to assess the stability of neuropsychological data from this population. Three studics utilizcd repeated testing (Cernovsky, 1984; Grant et al., 1978; Rounsaville, et al., 1982). However, Rounsaville et al. only examined their data for improvement effects resulting from a treatment intervention, and no attempt was made to assess the consistency between test and retest scores. Consistency was investigated by Cernovsky and by Grant et al., although the interpretation of their results is limited. Cernovsky used only a single dependent measure (the Clarke-WAIS vocabulary score), and Grant et al. compared only global impairment ratings between sessions and not the individual test scores on which these ratings were based.

The present study, therefore, assessed the stability of a broad range of neuropsychological scores by employing a test-retest design, in the absence of an intervening treatment protocol and with a brief interval between test sessions. Stability estimates were based on intraclass correlations between pairs of tests. These coefficients were then compared to those from previous studies employing identical or similar tests with non-drug-users.

\section{METHOD}

\section{Subjects}

Sixteen parenteral drug users (13 male and 3 female) were individually recruited. Seven subjects were currently enrolled in an outpatient methadone maintenance program, and the remainder were untreated polydrug users. Participation was voluntary, and all subjects received an hourly remuneration. Based on information provided during the initial session, ages ranged from 31 to 57 with a mean of 39.5 years. Education ranged from 9 to 14 years, with a mean of 11.4 years. Nine subjects $(56 \%)$ were either high school graduates or had received the equivalent of a highschool education. One subject had attended 2 years of college. All but one subject were right handed.

\section{Design}

All subjects were tested twice. Due to scheduling difficulties, the interval between the sessions ranged from 3 to 39 days, with a mean of 10.4 days. Four trained 
neuropsychological examiners were employed and subjects were randomly assigned to these examiners. To assess whether test-retest stability was examiner-independent, test and retest sessions were conducted by different examiners for each subject.

\section{Materials}

The following battery of neuropsychological tests was administered during both sessions in a standard order:

1. The Modified Mini-Mental State Examination (mMMS: Stern, Sano, Paulson \& Mayeux, 1987) was given as a test of general intellectual function. This test incorporates the digit-span subtest of the WAIS.

2. The Conceptual Levels Analogies Test (CLAT: Wilner, 1970) was employed to assess abstract reasoning. The first half of the tests, consisting of 21 multiple-choice verbal analogies, was used.

3. The Selective Reminding Test (SRT: Buschke \& Fuld, 1974). This is a test of verbal memory, consisting of a 12-item free-recall word list (Form 3 for both initial and retest), repeated 6 times, with the subject being reminded of item omissions only at the end of each trial. Four subscores are utilized; the total words recalled, long-term recall (LTR), long-term storage (LTS), and consistent long-term recall (CLTR). This test also includes a 15-minute delayed recall task, with an additional multiplechoice recognition task for words not recalled.

4. The Boston Naming Test (Kaplan, Goodglass, \& Weintraub 1983) was used to assess the ability to name visually depicted objects. Administration of this test was abbreviated by use of every other item on the list for a total of $\mathbf{3 0}$ target pictures.

5. The Controlled Oral Word Association Test (COWAT: Benton \& Hamsher 1978) measured verbal fluency by counting the number of words generated by the subject beginning with the letters $C$, $F$, and $L$, with a time limit of 60 seconds for each letter.

6. The Animal Naming subtest of the Boston Diagnostic Aphasia Examination (Goodglass \& Kaplan 1983) assessed the ability of the subject to generate animal names from memory while under a time constraint.

7. The Odd-Man Out Test (Flowers \& Robertson 1985). This test was used to investigate executive or "frontal lobe" function by assessing the ability of subjects to switch set. The subject was asked to choose a selection rule to determine which of three symbols (either shapes or letters) did not belong with the other two and to maintain that rule over a set of 10 triad presentations. The subject was then required to systematically alternate between two selection rules over three further sets of triads. A total correct score, summed over the four trials, was calculated.
8. The Benton Line Orientation Test (Benton, Hamsher, Varney, \& Spreen, 1983) was used to examine visuospatially function.

9. Two cancellation tasks, one utilizing a shape (diamond) and another a letter triad (TMX) were employed to assess attention (Sano, Rosen and Mayeux 1984).

10. The Digit Symbol subtest of the WAIS-R battery (Wechsler, 1981) also served as a measure of attention.

11. The Trail Making Test (Reitan \& Wolfson 1985) was administered as a screen test as well as a test of speeded performance and, in part B, a measure of the ability for rapid sequencing and set shifting.

12. The Perdue Pegboard (Tiffin 1968) was used to test manipulative (finger) dexterity and speed. The three placing tasks - dominant hand, nondominant hand and both hands - were used, with two trials per task administered during each session and a mean score for the two trials derived for each task.

Baseline test-retest coefficients for these tests, where available, are summarized in Table 1 .

\section{Procedure}

All subjects were tested individually in a quiet room (an office or examining room) free from interruption. Preliminary demographic information (age, handedness, current occupation, education, and first language spoken) was obtained at the beginning of both sessions. Prior to testing, each subject was asked to indicate what drugs he or she had taken during the past 24 hours. Regardless of reply, the use of specific drugs, including heroin, cocaine, crack, marijuana, and alcohol, was queried.

\section{Analysis}

In order to assess test-retest reliability, intraclass correlation coefficients (ICCs) were calculated between pairs of test scores, using equation $\operatorname{ICC}(3,1)$ recommended by Shrout and Fleiss (1979), which treats repeated tests as analogous to fixed raters in a generalizability $(\mathrm{G})$ study. The significance of these coefficients was tested using the $F$ ratio as indicated by these authors. In addition, between-session changes in the test scores was investigated using paired $t$ tests for scores with a normal or quasi-normal distribution and Wilcoxon matchedpairs signcd-ranks tests where test scores were markedly skewed or lacked a meaningful distribution.

\section{RESULTS}

\section{Demographic Data}

Information concerning age, handedness, and first language spoken was consistent over the two sessions. 
TABLE 1

Summary of Baseline Test-Retest Reliability Studies for the Neuropsychological Test Used

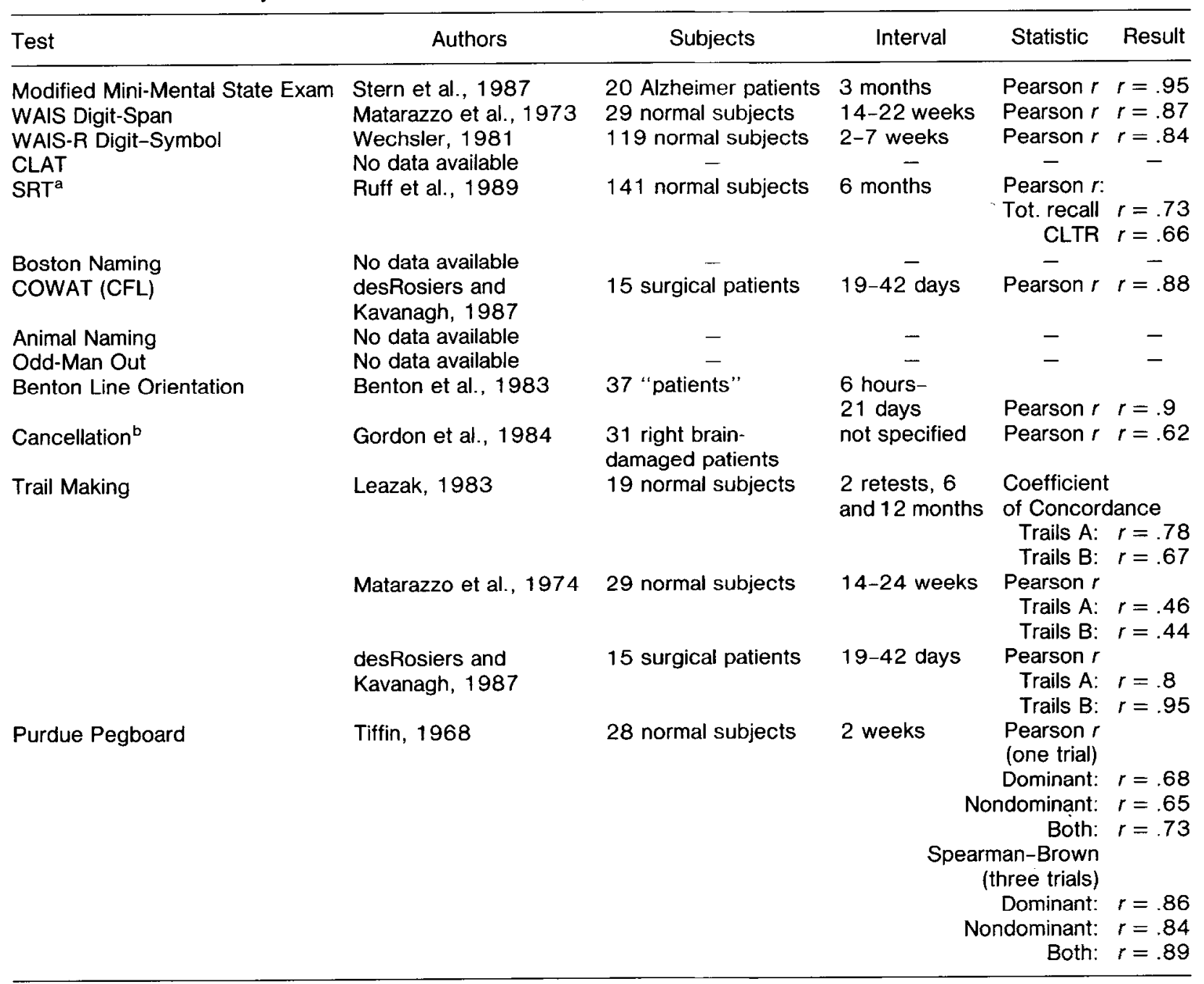

aUsing Form I for initial test and Form II for retest.

Based on $\mathrm{C}$ and $\mathrm{E}$ targets: no data available for TMX and shape task.

However, two discrepancies between sessions in the reported number of years of education were noted. One subject reported 2 years of college education and another subject claimed college graduation at the second test session, whereas both subjects had reported only high school education at the initial session.

\section{Current Drug Toxicology}

None of the 7 subjects currently enrolled in an outpatient methadone maintenance program reported the use of any drug other than methadone prior to either test session. Of the remaining subjects, 7 reported illicit drug use (either heroin, cocaine, or crack) within 24 hours of at least one of the two test sessions. Of these 7,4 reported drug use prior to both sessions. Only 2 subjects reported no drug use at all prior to either session.

\section{Neuropsychological Data}

Means and standard deviations for the neuropsychological test scores during both test sessions, along with the ICC for each pair of scores, are presented in Table 2. As a result of floor effects, ICCs for the cancellation task commission errors were not obtained. Similarly, the delayed recognition subtest of the SRT was omitted from the analysis because of a ceiling effect.

It can be seen that coefficients for most tests exceeded .5, and many were substantially higher than this. However, two tests failed to show a reasonable degree of stability. These were the SRT, where only the number of different intrusions showed anything more than a marginally significant correlation between sessions, and all three conditions of the Perdue Pegboard. Subjects enrolled in a methadone program showed significantly better neuropsychological performance (during 
TABLE 2

Means, Standard Deviations, and Intraclass Correlation Coefficients for the Initial and Retest Neuropsychological Measures

\begin{tabular}{|c|c|c|c|c|c|c|}
\hline \multirow[b]{2}{*}{ Test } & \multicolumn{2}{|c|}{ Initial } & \multicolumn{2}{|c|}{ Retest } & \multirow[b]{2}{*}{$\mathrm{ICC}^{\mathrm{a}}$} & \multirow[b]{2}{*}{$p$} \\
\hline & Mean & $(S D)$ & Mean & $(S D)$ & & \\
\hline \multicolumn{7}{|l|}{ WAIS Digit Span: } \\
\hline Total & 10.44 & $(2.58)$ & 10.81 & $(2.4)$ & .754 & $<.001$ \\
\hline Forwards & 5.94 & $(1.53)$ & 6.25 & $(1.34)$ & .718 & $<.001$ \\
\hline Backwards & 4.5 & $(1.63)$ & 4.56 & $(1.63)$ & .613 & $<.005$ \\
\hline CLAT & 9.67 & $(5.64)$ & 11.53 & $(4.44)$ & .814 & $<.001$ \\
\hline \multicolumn{7}{|l|}{ Selective Reminding Test: } \\
\hline Total recall & 49.81 & $(8.64)$ & 56.44 & $(8.24)$ & .42 & $<.05$ \\
\hline LTR & 40.62 & $(12.75)$ & 48.81 & $(12.91)$ & .395 & $<.05$ \\
\hline LTS & 43.62 & $(12.47)$ & 51.38 & $(11.94)$ & .303 & NS \\
\hline CLTR & 33.19 & (13.95) & 41.44 & (16.83) & .429 & $<.05$ \\
\hline Intrusions & 1.25 & $(1.06)$ & 0.81 & $(1.22)$ & .596 & $<.005$ \\
\hline Delayed Recall & 7.56 & $(2.78)$ & 8.81 & $(2.34)$ & .309 & NS \\
\hline Delayed Recognition & 11.81 & $(0.4)$ & 12.0 & (0) & - & - \\
\hline Boston Naming Test & 22.56 & $(3.2)$ & 23.94 & (3.34) & .876 & $<.001$ \\
\hline COWAT & 33.4 & (9.15) & 37.0 & $(9.72)$ & .694 & $<.001$ \\
\hline Animal Naming & 19.6 & (6.21) & 19.31 & $(5.8)$ & .827 & $<.001$ \\
\hline Benton Line Orientation & 20.62 & (4.69) & 21.88 & $(5.08)$ & .647 & $<.005$ \\
\hline Odd-Man Out (total correct) & 34.56 & $(7.43)$ & 33.75 & $(4.6)$ & .532 & $<.025$ \\
\hline \multicolumn{7}{|l|}{ Letter Triad Cancellation } \\
\hline Time (seconds) & 58.62 & $(18.72)$ & 56.75 & $(17.92)$ & .908 & $<.001$ \\
\hline Omission errors & 1.13 & (2.53) & 1.25 & $(2.52)$ & .97 & $<.001$ \\
\hline \multicolumn{7}{|l|}{ Shape Cancellation } \\
\hline Time (seconds) & 48.0 & $(10.03)$ & 46.62 & $(13.77)$ & .738 & $<.001$ \\
\hline Omission errors & 2.19 & $(2.17)$ & 2.38 & $(1.78)$ & .675 & $<.001$ \\
\hline WAIS-R Digit Symbol (AgeSc) & 7.75 & $(2.54)$ & 8.19 & $(2.9)$ & .964 & $<.001$ \\
\hline \multicolumn{7}{|l|}{ Trails A } \\
\hline Time & 42.06 & $(14.27)$ & 39.75 & $(14.21)$ & .764 & $<.001$ \\
\hline Errors & 0 & (0) & 0.12 & $(0.34)$ & - & - \\
\hline \multicolumn{7}{|l|}{ Trails B } \\
\hline Time & 105.79 & $(42.98)$ & 98.73 & $(40.44)$ & .644 & $<.005$ \\
\hline Errors & 1.07 & $(1.49)$ & 0.47 & $(0.74)$ & - & - \\
\hline \multicolumn{7}{|l|}{ Perdue Pegboard } \\
\hline Dominant & 13.5 & $(1.45)$ & 14.28 & $(1.9)$ & .018 & NS \\
\hline Nondominant & 12.7 & $(1.25)$ & 13.0 & (1.79) & .411 & $<.05$ \\
\hline Both & 9.93 & $(0.98)$ & 10.19 & $(1.78)$ & .391 & NS \\
\hline
\end{tabular}

antraclass correlation coefficient.

one or both sessions) than the polydrug users on several tests, including Trail Making (Parts A and B), the CLAT, and Animal Naming. Performance differences between subjects who reported drug use prior to one session versus both sessions were negligible.

Several scores showed a significant improvement from the initial to the retest session. These were the CLAT $(t[14]=-2.34, p=.035)$, the SRT total scorc $(t[15]=-2.91,=15, p=.011)$, the SRT LTR score $(t[15]=-2.32, p=.035)$, the SRT LTS score $(t[15]=$ $-2.15, p=0.48)$, the Boston Naming Test $(t[15]=$ $-3.38, p=.004)$ and the age-scaled Digit-Symbol subtest of the WAIS-R $(t[15]=-2.41, p=.029)$. In addition, the COWAT score showed a marginal improvement between sessions $(t[14]=-2.11, p=.053)$. These improvements are likely to be practice effects. Finally, in order to assess the effect of the varying interval between the two test sessions (see Design), neuropsychological change scores were calculated by subtracting each initial test score from its corresponding retest value. These change scores were then correlated with the test-retest interval (days). Only two correlations were significant - with the CLAT change score $(r=.58, p=.011)$ and with the change in the number of TMX cancellation omissions $(r=.77, p<.001)$. In both cases, longer test-retest intervals were associated with greater change scores.

\section{DISCUSSION}

Data obtained from the sample of drug abusers in the present study reached an acceptable standard of reliability. Most of the demographic information given the initial session was consistently repeated in the second 
session. The reliability of this information corroborates previous research. For example, McLellan et al. (1985) found high test-retest reliability for drug abusers with responses to the Addiction Severity Index, a structured interview designed to assess problem severity in seven areas: medical condition, employment, drug use, alcohol use, illegal activity, family relations, and psychiatric condition. Moreover, demographic data obtained from drug abusers has been shown to have good crossreliability. For example, Ball (1967) found an $82.8 \%$ agreement rate and Stimson (1973) found a $96 \%$ agreement rate between self-reported age and age according to case notes for narcotic addicts. However, discrepancies in reported education level were found between sessions in the present study. Since the scores of several neuropsychological tests are adjusted for education for the purpose of evaluation, these discrepancies highlight the need for circumspection when obtaining personal data from this population.

Turning to the neuropsychological data itself, a high degree of test-retest stability was found for most of the tests administered. Since $\operatorname{ICC}(3,1)$ and the Pearson correlation both produce coefficients with similar values (Shrout, personal communication, 1989), a direct comparison between the present results and the baseline coefficients reported in Table 1 was possible. Coefficients for the mMMS, WAIS Digit-span, and both Trail Making tests were of similar magnitude to the those reported in this table. Indeed, two tests (WAIS-R Digit-Symbol and the Cancellations) showed higher coefficients than corresponding baseline values. Some correlations (the COWAT and Benton Line Orientation) were notably lower than those found with non-drug-users, but remained within comfortable limits nevertheless. Only two tests showed unacceptably low stability. These were the SRT and the Perdue Pegboard. Reasons for this instability are unclear.

In both cases, it is unlikely to have resulted from the tests themselves. Table 1 indicates stability coefficients of high magnitude for the Perdue Pegboard with nondrug-users, results that are corroborated by data from an earlier version of this test (see Tiffin 1968). Only one normative test-retest study has been carried out on the SRT (Ruff, Light, \& Quayhagen, 1989), showing good reliability (although these authors used two different word lists rather than repeating the same list). It is reasonable to assume, therefore, that subject characteristics and/or state were significant contributors to the instability found in these two tests. For example, verbal memory and manual dexterity may be particularly sensitive to phasic drug effects. However, the effects of acute drug toxicity were difficult to investigate in the present study, partly because most subjects reported drug use prior to both sessions and also because the sample size was too small to permit alternative stratified analyses (e.g., methadone versus untreated users).

Alternatively, it may be that performance with the
SRT and Perdue Pegboard is vulnerable to subtle shifts in attention and concentration. It should be noted, however, that two explicit tests of attention - the DigitSpan and Digit-Symbol subtests of the WAIS - were found to be highly stable in this study. Nor is it likely that the instability in the SRT and the Perdue Pegboard was caused by fluctuations in compliance and/or motivation, since such fluctuations would be expected to exert a global effect across the test battery, rather than to cause the selective disturbances observed here. Further investigations of the stability of verbal memory and motor dexterity in drug abusers are clearly warranted.

In summary, while there are several reasons to anticipate a high degree of variability and instability in the neuropsychological performance of drug abusers, the present study found an acceptable level of consistency in a wide range of neuropsychological tests using a test-retest paradigm with a heterogeneous group of drug users. The battery administered included tests of general intellectual function, abstract reasoning, verbal memory, language attention, visuospatial ability, set switching, speeded performance, and manipulative dexterity. High reliability coefficients were found with these tests, with the exception of the Selective Reminding Test and the Perdue Pegboard. Reasons for poor stability with these two tests are unclear, however, and further reliability studies would be provident. In the meantime, the inclusion of alternative and/or supplementary memory and motor tests are recommended for neuropsychological investigations with this population.

\section{REFERENCES}

Ball, J.C. (1967). The reliability and validity of interview data obtained from 59 narcotic drug addicts. American Journal of Sociology, 72, 650-654.

Benton, A., \& Hamsher, K. de S. (1978). Multilingual Aphasia Examination. Iowa City: University of Iowa Hospitals.

Benton, A., Hamsher, K. de S., Varney, N., \& Spreen, O. (1983). Contributions to neuropsychological assessment. New York: Oxford University Press.

Brown, R.R., \& Partington, J.E. (1942). A psychometric comparison of narcotic addicts with hospital attendants. Journal of General Psychology, 27, 71-79.

Buschke, H., \& Fuld, P.A. (1974). Evaluating storage, retention and retrieval in disordered memory and learning. Neurology, 24, 1019-1025.

Chaney, E.F., Roszell, D.K., \& Cummings, C. (1982). Relapse in opiate addicts: A behavioral analysis. Addictive Behaviors, 7, 291-297.

Cernovsky, Z. (1984). Stability of Clarke-WAIS vocabulary scores in chronic alcoholics and drug addicts. Ontario Psychologist, 16, 11-12.

desRosiers, G., \& Kavanagh, D. (1987). Cognitive assessment in closed head injury: Stability, validity and parallel forms for two neuropsychological measures of recovery. International Journal of Clinical Neuropsychology, 9, 162-173.

American Psychiatric Association. (1987). Diagnostic and statistical manual of mental disorders (3rd ed., rev.). Washington, DC: Author. 
Flowers, K., \& Robertson, C. (1985). The effect of Parkinson's disease on the ability to maintain a mental set. Journal of Neurology, Neurosurgery and Psychiatry, 48, 517-529.

Goodglass, H., \& Kaplan, D. (1983). The assessment of aphasia and related disorders (2nd ed.). Philadelphia: Lea and Febiger.

Gordon, W.A., Ruckdeschel-Hibbard, M., Egelko, S., Diller, L., Simmens, S., Langer, K., Sano, M., Orazem, J., \& Weinberg, J. (1984). Evaluation of the deficits associated with right brain damage: Normative data on the Institute of Rehabilitation Medicine Test Battery. Research and Training Center on Head Trauma and Stroke, New York University Medical Center.

Grant, I., Adams, K.M., Carlin, A.S., Rennick, P.M., Judd, L.L. \& Schooff, K. (1978). The collaborative neuropsychological study of polydrug users. Archives of General Psychiatry, 35, 1063-1074.

Guerra, D., Sole, A., Cami, J., \& Tobena, A. (1987). Neuropsychological performance in opiate addicts after rapid detoxification. Drug and Alcohol Dependence, 20, 261-270.

Judson, B.A., \& Golstein, A. (1983). Episodes of heroin use during maintenance treatment with stable dosage of $(-)$-a-acctylmethadol (methadyl acetate, LAAM). Drug and Alcohol Dependence, 11, 271-278.

Kaplan, E., Goodglass, H., \& Weintraub, S. (1983). The Boston Naming Test. Philadelphia, PA: Lea and Febiger.

Leazak, M.D. (1983). Neuropsychological assessment. New York: Oxford University Press.

Matarazzo, J.D., Weins, A.N., Matarazzo, R.G., \& Goldstein, S.G. (1974). Psychometric and clinical test-retest reliability of the Halstead-Reitan impairment index in a sample of healthy young normal men. Journal of Nervous and Mental Disease, 158, 37-49.

Matarazzo, R.G., Wiens, A.N., Matarazzo, J.D., \& Manaugh, T.S. (1973). Test-retest reliability of the WAIS in a normal population. Journal of Clinical Psychology, 29, 195-197

McLellan, A., Luborsky, L., Cacciola, J., Griffith, J., Evans, F., Barr, H., \& Obrien, C. (1985). New data from the Addiction Severity Index: Reliability and validity in three centers. Journal of Nervous and Mental Disease, 173, 412-423.

Meyer, R.E., \& Hesselbrock M.N. (1984). Psychopathology and addictive disorders revisited. In Mirin S.M. (Ed.), Substance abuse and psychopathology. Washington, DC: American Psychiatric Press.

Reitan, R.M., \& Wolfson, D. (1985). The Halstead-Reitan Neuropsychological Test Battery: theory and clinical interpretation. Tuscon, AZ: Neuropsychology Press.
Ross, H.E., Glaser, F.B., \& Germanson, T. (1988). The prevalence of psychiatric disorders in patients with alcohol and other drug problems. Archives of General Psychiatry, 45, 1023-1031.

Roszell, D.K., \& Calsyn, D.A. (1986). Methadone dosage: Patient characteristics and clinical correlates. International Journal of the Addictions, 21, 1233-1246.

Rounsaville, B.J., Weissman, M.M., Kleber, H., \& Wilber, C.H. (1982). The heterogeneity of psychiatric disorders in opiate addicts. Archives of General Psychiatry, 39, 161-166.

Rounsaville, B.J., Jones, C., Novelly, R.A., \& Kleber, H. (1982). Neuropsychological functioning in opiate addicts. Journal of Nervous and Mental Diseases, 170, 209-216.

Ruff, R.M., Light, R.H., \& Quayhagen, M. (1989). Selective Reminding Test: A normative study of verbal learning in adults. Journal of Clinical and Experimental Neuropsychology, 11, 539-550.

Sano, M., Rosen, W., \& Mayeux, R. (1984, August). Attention deficits in Alzheimer's disease. Paper presented at the 92nd Annual Meeting of the American Psychological Association, Toronto, Ontario, Canada.

Shrout, P.E., \& Fleiss, J.L. (1979). Intraclass correlations: Uses in assessing rater reliability. Psychological Bulletin, 86, 420-428.

Stern, Y., Sano, M., Paulson, J., \& Mayeux, R. (1987). Modified Mini-Mental State Examination: Validity and reliability. Neurology, 37, 179.

Stimson, G.V. (1973). Heroin and behavior. New York: Halstead Press: Wiley.

Tiffin, J. (1968). Perdue Pegboard: Examiner manual Chicago: Science Research Assoc.

Wagner, E.E., \& Caldwell, M.S. (1979). WAIS test-retest reliability for a clinical out-patient sample. Perceptual and Motor Skills, 48, 131-137.

Wechsler, D. (1981). Manual of the Wechsler Adult Intelligence ScaleRevised. New York: The Psychological Corporation, Harcourt Brace Jovanovich.

Weiss, R.D., \& Mirin, S.M. (1984). Drug, host, and environmental factors in the development of chronic cocaine abuse. In S.M. Mirin (Ed.), Substance abuse and psychopathology. Washington, DC: American Psychiatric Press.

Wilner, A.E. (1970). Towards development of more sensitive clinical tests of abstraction: the analogy test. Proceedings of the Annual Convention of the American Psychological Association, 5 , 553-554. 